\title{
Usefulness of open mixed nut challenges to exclude tree nut allergy in children
}

\author{
Francine C. Van Erp ${ }^{1 *}$, André C. Knulst ${ }^{3}$, Irene L. Kok ${ }^{2}$, Maartje F. van Velzen ${ }^{1}$, Cornelis K. van der Ent ${ }^{1}$ \\ and Yolanda Meijer ${ }^{1}$
}

\begin{abstract}
Background: To minimize the risk of accidental reactions, atopic children with multiple sensitizations to tree nuts are advised to avoid all nuts. Multiple food challenges would be needed to confirm the clinical relevance, but are too burdensome to be practical.

The usefulness of open mixed nut challenges in terms of safety, reactions during challenge, tolerance of the challenge material, effect on the elimination diet and satisfaction of the parents was evaluated.

Findings: Open mixed nut challenges were performed in 19 children with a previous negative hazelnut challenge and long term elimination diet for tree nuts. Challenges were negative in 13 (68\%) children, in four (21\%) children (non-severe) allergic symptoms were observed. The challenges were well accepted, safe and efficient. We were able to avoid multiple nut challenges in 15 (79\%) children.
\end{abstract}

Conclusions: Open mixed nut challenge can efficiently exclude multiple tree nut allergies in children with a lifelong nut free diet and low suspicion of clinical allergy.

Keywords: Tree nuts, Nut allergy, Diagnostics, Food challenge

\section{Findings}

Suspected food allergy based on sensitisation without known ingestion often results in elimination diets and subsequent social isolation, fear of anaphylaxis and a decreased quality of life [1]. In atopic children tree nut elimination diets are often advised due to multiple tree nut sensitisations together with severe eczema or other (severe) food allergies $[2,3]$. In those children introduction at home is often not possible due to the possible risk of allergic reactions and fear of the parents. The Double Blind Placebo Controlled Food Challenge (DBPCFC) is the gold standard to diagnose food allergy [4]. In children presenting with a (life) long elimination diet for tree nuts, multiple challenges would be necessary to rule out the presence of relevant tree nut allergies. This is however difficult in practice as DBPCFCs are time consuming, expensive and burdensome procedures. Open mixed nut challenges have previously been shown to efficiently

\footnotetext{
* Correspondence: f.c.vanerp@umcutrecht.nl

'Department of Paediatric Pulmonology and Allergology, Wilhelmina Children's Hospital, University Medical Centre Utrecht, 3508 AB, P O Box 85090, Utrecht, The Netherlands

Full list of author information is available at the end of the article
}

discriminate between multiple tree nut allergies and a single nut allergy [5]. In this study we describe our experience with open mixed nut challenges as a diagnostic tool to exclude multiple nut allergies in children with long term elimination diets for tree nuts.

We performed a retrospective case note review of food challenges performed in our tertiary food allergy clinic from 2012-2014 and selected children who underwent a mixed nut challenge. Data were obtained as part of regular clinical care, collected from electronic patient records by their responsible clinician and used in strictly anonymous form, according to the code of conduct for medical research approved by the hospital's Medical Ethical Committee.

Nineteen children with a previous negative hazelnut challenge and a lifelong preventive elimination diet for multiple tree nuts underwent a mixed nut challenge as part of clinical care. Children without a history of tree nut related symptoms and with low levels of sIgE to all tree nuts were challenged with six nuts (almond, walnut, cashew, pistachio, pecan, Brazil nut) (protocol A), Children with suspected cashew/pistachio allergy (previous symptoms or a specific $\mathrm{IgE}$ to cashew or pistachio of $>10 \mathrm{kU} / \mathrm{L}$ ) were challenged with four nuts (almond, walnut, pecan, Brazil nut) (protocol 
B). In short, $5 \mathrm{~g}$ of each tree nut was blended and mixed with apple sauce. Increasing amounts of the mixture were given in an open challenge with 21 grams of whole tree nuts as the last step in the protocol (Table 1). Challenges were discontinued and considered positive in case of objective symptoms or if suggestive subjective symptoms occurred at 3 subsequent doses or a subjective symptom lasted for more than 45 minutes. In case of a negative challenge parents were advised to introduce all tested tree nuts subsequently into the diet of their child. After positive or inconclusive challenges an expert team of allergists decided whether (guided) reintroduction of tree nuts was possible or future multiple single nut challenges were indicated at the patient's request. One month after challenge parents were contacted by phone to evaluate the reintroduction of tree nuts, dietary restrictions and satisfaction about the challenge procedures.

Measurement of specific IgE (sIgE) was performed in all children within 1 year prior to the food challenge, using Immuno CAP-technique (Phadia, Uppsala, Sweden), IgE levels of $\geq 0.35 \mathrm{kU} / \mathrm{L}$ were considered positive. The presence of asthma, atopic dermatitis, allergic rhinitis and other food allergies was determined in out-patient clinic consultations before food challenge. Reactions during the challenge, tolerance of the nut mix challenge material, and satisfaction of the parents were evaluated with descriptive statistics.

Children who underwent the mixed nut challenge had a mean age of 10.1 (range: 5.4-17.1) years. Elimination diets for tree nuts were based on (multiple) sensitizations together with known other food allergy in 14 (74\%) or severe eczema in $3(16 \%)$ children. Two other children (12\%) eliminated tree nuts based on a history of immediate symptoms after ingestion of cashew or pistachio.

Table 1 Challenge protocol of mixed nut challenges

\begin{tabular}{|c|c|c|c|c|}
\hline & & & $\begin{array}{l}\text { Mixture } \\
A^{a}\end{array}$ & $\begin{array}{l}\text { Mixture } \\
\mathrm{B}^{\mathrm{b}}\end{array}$ \\
\hline Portion & $\begin{array}{l}\text { Time } \\
\text { (min) }\end{array}$ & Mixture dose (g) & $\begin{array}{l}\text { Tree nuts } \\
\text { dose (g) }\end{array}$ & $\begin{array}{l}\text { Tree nuts } \\
\text { dose (g) }\end{array}$ \\
\hline 1 & 0 & 1 & 0,2 & 0,1 \\
\hline 2 & 15 & 4 & 0,7 & 0,5 \\
\hline 3 & 30 & 11 & 2,2 & 1,5 \\
\hline 4 & 60 & 33 & 6,7 & 4,4 \\
\hline 5 & 90 & 100 & 20,1 & 13,3 \\
\hline \multirow[t]{2}{*}{6} & 150 & $\begin{array}{l}\text { Open challenge with whole } \\
\text { nuts ( } 2 \text { almonds, } 2 \text { half walnuts, } \\
2 \text { half pecans, } 1 \text { Brazil nut, } 2 \\
\text { cashew nuts*, } 2 \text { pistachios*) }\end{array}$ & ca. 21 & ca. 17 \\
\hline & & Total & 51 & 37 \\
\hline
\end{tabular}

\footnotetext{
* Only in mixture A
}

${ }^{a}$ Mixture A: $5 \mathrm{~g}$ of each nut (almond, walnut, cashew, pistachio, pecan, Brazil nut) and $120 \mathrm{~g}$ apple sauce.

${ }^{\mathrm{b}}$ Mixture B: $5 \mathrm{~g}$ of each nut (almond, walnut, pecan, Brazil nut) and $130 \mathrm{~g}$ apple sauce.
Challenges were performed with mixture B in four children. Individual characteristics and sensitization patterns of all children are shown in Table 2 .

In 13 children $(68 \%)$ the mixed nut challenge was considered negative. Allergic symptoms were observed in $4(21 \%)$ children, all with Sampson grade 3 reactions [6]. No correlation between levels of sIgE and outcome of the mixed nut challenge could be found. In one positive tested child (case 3), IgE negative nuts were introduced successfully. In one other child (case 4) parents successfully introduced almond and walnut based on their own decision. In $2(11 \%)$ young children the test was inconclusive due to aversion. In one of those children (case 2) tree nuts with negative sIgE results were successfully introduced at home without additional challenges. Overall we were able to avoid multiple challenges in $15(79 \%)$ children and successfully could expand the diet in 14 (74 \%) children (Table 2). All parents were satisfied about the challenge material, challenge protocol and outcome of the challenge.

We successfully performed mixed nut challenges in children with previous negative hazelnut challenge and low suspicion of (multiple) tree nut allergy. Hazelnut allergy was ruled out first because sIgE to hazelnut was relatively high (mean $24.5 \mathrm{kU} / \mathrm{L}$ ). Moreover, hazelnut is one of the most frequently consumed tree nuts in Europe and the most prevalent cause of tree nut allergy in the Netherlands [7]. No clear cut-off point of sIgE to tree nuts was used to select children for mixed nut challenge as the results of the few previous published studies on this topic differ widely between patient populations and tree nuts $[8,9]$. However, children were never challenged when any sIgE level to tree nuts was above $10 \mathrm{kU} / \mathrm{L}$. The lack of correlation between sIgE values and mixed nut challenge outcome in our study demonstrates that the usefulness of multiple allergen testing in children with lifelong elimination diets is questionable. In children without multiple tree nut sensitization and without previous symptoms after ingestion, introduction at home is justified [4]. Nevertheless, in case of extreme anxiety in children and/or parents, mixed nut challenges were useful and successfully performed for this reason in three children (cases 7,13 and 17). Children with a clear history of tree nut related symptoms were excluded or not tested for cashew/ pistachio as they were expected to develop symptoms during the mixed nut challenge and therefore were unlikely to benefit from the mixed nut challenge in terms of accurate diagnosis and opportunities to expand the diet. In those children single nut challenges with nuts relevant to the child's diet were performed at parent's request. When symptoms occurred during challenge the true diagnostic value of the mixed nut challenge remained questionable as the culprit nut was unknown. However, as proposed by Ball et al. previously, 
Table 2 Children who underwent a mixed nut challenge

Case Age Reasonforlong Mix Max Symptoms

Outcome Conclusion \& advice

diet diet

Tree nut allergy,

multiple challenges

No No pain, vomiting and peanut allergy

13,3 Aversion

Multiple sensitization
and reaction to 1 nut (Cas S2)

$3 \quad 15,2$ Multiple sensitizationand eczema

$4 \quad 9,7$ Multiple sensitization A and peanut allergy

59 9,4 Multiple sensitization A and other food allergy

$6 \quad 5,4$ Multiple sensitization A and other food allergy

8 Multiple sensitization A and other food allergy

$8 \quad 11,4$ Multiple sensitization B and reaction to 1 nut (Pis S5)

$9 \quad 6,3$ Multiple sensitization B and other food allergy

$10 \quad 15,4$ Multiple sensitization A and peanut allergy

1110 Multiple sensitization A and peanut allergy

129 Multiple sensitization A and peanut allergy

139,8 Multiple sensitizationand eczema

$14 \quad 9,7$ Multiple sensitization A and peanut allergy

158 Multiple

sensitizationand eczema

20,1 Rhino-conjunctivitis

6,7 Rash, urticaria, conjunctivitis, sensation of throat tightness, abdominal pain

2,2 OAS, rhino-conjunctivitis, vomiting

6,7 Rash, rhino-conjunctivitis,

angioedema, vomiting

(n)

Introduction all nuts

Introduction all nuts

Introduction all nuts

Introduction all nuts

In, bra, pec, wa

bra

alm, bra, pec

ree nut allergy,

Tree nut allergy,

nuts war

multiple challenges

No No

Tree nut allergy,

multiple challenges

Introduction all nuts

nuts

nuts

nuts

nuts
Introduction all nuts

Alm Alm,

Alm,

wal $^{1}$

Suspected tree nut No Alm,

allergy, introduction of nuts bra,

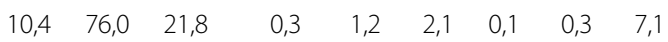

$\operatorname{pec}^{1}$

No Alm,

$\begin{array}{lllllllll}19,6 & 37,0 & 15,5 & 2,7 & 7,9 & 7,8 & 1,5 & 0,3 & 1,2\end{array}$

nuts nuts

$\begin{array}{lllllllll}50,0 & 101,0 & 74,0 & 0,4 & 2,7 & 4,1 & 0,2 & 0,3 & 0,3\end{array}$

Alm Alm

Alm None

$1,7 \quad 5,0 \quad 2,3$

$\begin{array}{llllll}0,2 & 2,3 & 3,3 & 0,0 & 0,5 & 0,6\end{array}$

$\begin{array}{lllllllll}37,0 & 73,0 & 32,8 & 0,5 & 0,0 & 0,0 & 0,1 & 0,1 & 0,0\end{array}$

No

$0,0 \quad 0,0 \quad 2,0$

$\begin{array}{llllll}0,6 & 12,0 & 23,7 & 0,4 & 0,7 & 2,9\end{array}$

nuts $^{3}$

No

$14,1 \quad 101,0 \quad 19,7$

$\begin{array}{llllll}0,3 & 7,3 & 10,8 & 0,0 & 0,8 & 2,8\end{array}$

Ntn No

$\begin{array}{lllllllll}101,0 & 43,0 & 8,5 & 2,4 & 0,4 & 0,6 & 0,1 & 0,9 & 1,9\end{array}$

No None

No None

No None

No None

No No

nuts nuts ${ }^{6}$ 
Table 2 Children who underwent a mixed nut challenge (Continued)

$16 \quad 17,1 \quad$ Multiple sensitization A and peanut allergy

$17 \quad 14,1$ Multiple sensitization A and other food

$$
\text { allergy }
$$

1810 Multiple sensitization A and peanut allergy

$19 \quad 12,5$ Multiple sensitization B and peanut allergy
Introduction all nuts

Introduction all nuts

Introduction all nuts

Introduction tested nuts No No

nuts nuts

Ntn None

No None

nuts
Ntn None

$18.90 \quad 1,4$

$30,0 \quad 101,0$

3.50

2,3
1,8

$1,21,1$

$0,2 \quad 0,4$

Alm, almond; Bra, Brazil nut; Cas, cashew nut; Haz, hazelnut; Ntn, no traces of nuts; Pec, pecan; Pis, pistachio; Wal, walnut

* Maximum dose in total grams mixed tree nut

+ , positive; -, negative; + /-, inconclusive

Introduction started based on negative slgE results

${ }^{2}$ Introduction started by parents

${ }^{3}$ Introduction not started due to comorbidities of the child

${ }^{4}$ Introduction successful but preventive nut free diet due to possible cashew or pistachio allergy

${ }^{5}$ Oral allergy symptoms after whole nuts

${ }^{6}$ Introduction not started, parents are used to diet due to other food allergies 
it is debatable whether single nut challenges will change the outcome of advice in children with (multiple) tree nut allergy as products are often not labelled with individual nuts [5]. Moreover even after negative mixed nut challenge other reasons as other food allergies or habituation to the diet (patient 15) can prevent patients from introducing tree nuts. Nonetheless, our data show that most parents $(74 \%)$ do manage to introduce (single) nuts in the diet even when aversion and or allergic symptoms are present during mixed nut challenge.

When interpreting the results of this study some limitations have to be discussed. We performed mixed nut challenges in a small and selected patient population to exclude multiple tree nut allergies and diminish unnecessary dietary restrictions in children with long term elimination diets. Further research is necessary to determine whether mixed nut challenges are feasible in different populations. We did not have information on skin prick tests or sensitization to tree nut components. In the future, it might be possible to exclude tree nut allergy at least for some tree nuts based on component resolved diagnostics [10]. However, some parents will not introduce tree nuts at home because of fear for an allergic reaction, in those cases mixed nut challenge are still useful despite its limited diagnostic value. Previously published data provide evidence for a high degree of cross reactivity between walnut and pecan and cashew and pistachio $[11,12]$. As a consequence one could argue that mixed nut challenges can be simplified to contain four nuts only (cashew or pistachio, walnut or pecan, almond and Brazil nut). The challenge material of our mixed nut challenges is simple and can be easily implemented in daily practice and adapted to the individual patient. It is unclear whether the material in its present form is suitable for children below the age of six. As an alternative, a biscuit with mixed nuts was used successfully in a previous study [5]. This however requires a specialized kitchen and involves heating of the allergens which can reduce their allergenicity [13]. All parents were satisfied with the challenge, however in future research it would be important to compare our results with parents of children who underwent multiple single challenges.

In conclusion, our data show that open mixed nut challenges are useful to exclude multiple tree nut allergies in children in with a lifelong nut free diet and low suspicion of clinical allergy. In those children mixed nut challenges can prevent multiple single nut challenges and help to facilitate introduction of tree nuts at home, even when symptoms during challenge occur.

Abbreviations

DBPCFC: Double blind placebo controlled food challenge; SlgE: Specific IgE.

\section{Competing of interest}

All authors declare that they have no competing interests relevant to this manuscript.

\section{Authors' contributions}

FE substantially contributed to design, concept, acquisition of data, analysis and interpretation of data, drafting the article and final approval of version to be published. AK substantially contributed to interpretation of data, drafting the article and revising critically for important intellectual content and final approval of version to be published. IK, MV and CE substantially contributed to interpretation of data, revising critically for important intellectual content and final approval of version to be published. YM substantially contributed to design, interpretation of data and revising critically for important intellectual content and final approval of version to be published. All authors read and approved the final manuscript.

\section{Author details}

${ }^{1}$ Department of Paediatric Pulmonology and Allergology, Wilhelmina Children's Hospital, University Medical Centre Utrecht, 3508 AB, P O Box 85090, Utrecht, The Netherlands. ${ }^{2}$ Department of Dietetics, Internal Medicine and Dermatology, University Medical Centre Utrecht, Utrecht, The Netherlands. ${ }^{3}$ Department of (Paediatric) Dermatology and Immunlogy, University Medical Centre Utrecht, Utrecht, The Netherlands.

Received: 10 February 2015 Accepted: 4 May 2015

Published online: 16 May 2015

\section{References}

1. Flokstra-de Blok BMJ, EJ D a, Vlieg-Boerstra BJ, Oude Elberink JNG, Raat H, DunnGalvin A. Health-related quality of life of food allergic patients: comparison with the general population and other diseases. Allergy. 2010;65:238-44.

2. Chiu C-Y, Huang Y-L, Tsai M-H, Tu Y-L, Hua M-C, Yao T-C, et al. Sensitization to food and inhalant allergens in relation to atopic diseases in early childhood: A birth cohort study. PLoS One. 2014;9, e102809.

3. Mailhol C, Giordano-Labadie F, Lauwers-Cances V, Ammoury A, Paul C, Rance F. Point prevalence and risk factors for food allergy in a cohort of 386 children with atopic dermatitis attending a multidisciplinary dermatology/ paediatric allergy clinic. Eur J Dermatol. 2014;24:63-9.

4. Sampson H, van Wijk R G, Bindslev-Jensen C, Sicherer S, Teuber SS, Burks AW, et al. Standardizing double-blind, placebo-controlled oral food challenges: American Academy of Allergy, Asthma \& Immunology-European Academy of Allergy and Clinical Immunology PRACTALL consensus report. J Allergy Clin Immunol. 2012;130:1260-74.

5. Ball H, Luyt D, Bravin K, Kirk K. Single nut or total nut avoidance in nut allergic children: Outcome of nut challenges to guide exclusion diets. Pediatr Allergy Immunol. 2011;22:808-12.

6. Sampson HA. Anaphylaxis and emergency treatment. Pediatrics. 2003;111 (6 Pt 3):1601-8.

7. Jenab M, Sabaté J, Slimani N, Ferrari P, Mazuir M, Casagrande C, et al. Consumption and portion sizes of tree nuts, peanuts and seeds in the European Prospective Investigation into Cancer and Nutrition (EPIC) cohorts from 10 European countries. Br J Nutr. 2007;96:S12.

8. Ludman S, Ballabeni P, Eigennman PA, Wassenberg J. Predicting positive food challenges in children sensitised to peanuts/tree nuts. Pediatr Allergy Immunol. 2013;1-6.

9. Clark AT, Ewan PW. Interpretation of tests for nut allergy in one thousand patients, in relation to allergy or tolerance. Clin Exp Allergy. 2003;33:1041-5.

10. Roux K, Teuber S, Sathe S. Tree nut allergens. Int Arch allergy. 2003;131:234-44.

11. Willison LN, Tawde P, Robotham JM, Penney RM, Teuber SS, Sathe SK, et al. Pistachio vicilin, Pis $\vee 3$, is immunoglobulin E-reactive and cross-reacts with the homologous cashew allergen, Ana o 1. Clin Exp Allergy. 2008;38:1229-38.

12. Goetz DW, Whisman BA, Goetz AD. Cross-reactivity among edible nuts: double immunodiffusion, crossed immunoelectrophoresis, and human specific igE serologic surveys. Ann Allergy Asthma Immunol. 2005;95:45-52.

13. Masthoff $L$, Hoff $R$, Verhoeckx KCM, van Os-Medendorp $H$, MichelsenHuisman A, Baumert $J$, et al. A systematic review of the effect of thermal processing on the allergenicity of tree nuts. Allergy. 2013;68:983-93. 